\title{
Spin-lattice interaction in the insulator-to-metal transition of $\mathrm{GdBaCo}_{2} \mathrm{O}_{5+\delta}$
}

\author{
Mattia Allieta, ${ }^{1,}$ Cesare Oliva, ${ }^{1}$ Marco Scavini, ${ }^{1}$ Serena Cappelli, ${ }^{1}$ Ekaterina Pomjakushina, ${ }^{2}$ and Valerio Scagnoli ${ }^{3}$ \\ ${ }^{1}$ Dipartimento di Chimica Fisica ed Elettrochimica, Università degli Studi di Milano, Via Golgi 19, IT-20133 Milano, Italy \\ ${ }^{2}$ Laboratory for Developments and Methods, Paul Scherrer Institut, CH-5232 Villigen PSI, Switzerland \\ ${ }^{3}$ Swiss Light Source, Paul Scherrer Institut, CH-5232 Villigen PSI, Switzerland.
}

(Received 1 September 2011; revised manuscript received 2 December 2011; published 27 December 2011)

\begin{abstract}
We use electron paramagnetic resonance spectroscopy (EPR) and synchrotron radiation x-ray powder diffraction to study the temperature-induced insulator-to-metal transition (IMT) for $\mathrm{GdBaCo}_{2} \mathrm{O}_{5+\delta}$ samples in the $\delta$ range $0.54(1) \leqslant \delta \leqslant 0.63(1)$. The EPR linewidth markedly changes across IMT and its temperature evolution can be explained considering spin state transition involving Co ions. The temperature dependences of the EPR linewidth and of the $a$ lattice parameter fairly overlap each other suggesting spin-lattice interaction along the same crystallographic direction of the reported Ising-like spin anisotropy [A. A. Taskin et al., Phys. Rev. Lett. 90, 227201 (2003)]. A possible mechanism describing the interplay between this strong spin-lattice interaction and IMT is proposed.
\end{abstract}

DOI: 10.1103/PhysRevB.84.235144

PACS number(s): 71.30.+h, 76.30.-v, 61.05.cp, 61.66.Fn

\section{INTRODUCTION}

$L n \mathrm{BaCO}_{2} \mathrm{O}_{5+\delta}$ layered cobalt oxides ( $\mathrm{Ln}$ is a rare earth element) exhibit very rich electronic and magnetic phase diagrams that involve many fascinating phenomena such as the magnetoresistance (MR) effect. ${ }^{1-3}$ The crystal structure of $L n \mathrm{BaCo}_{2} \mathrm{O}_{5+\delta}$ can be viewed as a sequence of square-lattice layers $\left[\mathrm{CoO}_{2}\right][\mathrm{BaO}]\left[\mathrm{CoO}_{2}\right]\left[\mathrm{LnO} \mathrm{O}_{\delta}\right]$ stacking along the $c$ axis with alternation of two types of coordination environments for cobalt ions, i.e., $\mathrm{CoO}_{5}$ pyramid $\left(\mathrm{Co}_{\text {pyr }}\right)$ and $\mathrm{CoO}_{6}$ octahedra $\left(\mathrm{Co}_{\text {oct }}\right)$. The $\mathrm{Co}_{\mathrm{pyr}} / \mathrm{Co}_{\text {oct }}$ ratio as well as their ordering along crystallographic directions can be modified by tuning the oxygen concentration $\delta$ in $\mathrm{LnO}_{\delta}$ planes. ${ }^{2}$ More importantly, $\delta$ affects the mean valence state of cobalt ions as $\mathrm{Co}^{3+}$ can exist in low-, intermediate-, or high-spin states (LS, IS, or HS) while $\mathrm{Co}^{2+}$ and $\mathrm{Co}^{4+}$ are stable in HS and LS configurations, respectively. ${ }^{2}$ Then, the physics of these systems is driven by a complex interplay between charge, spin, orbital, and lattice degrees of freedom triggered, e.g., by temperature.

Such behavior is clearly demonstrated by the temperatureinduced insulator-to-metal transition (IMT) found at $T_{\mathrm{IM}} \approx$ $365 \mathrm{~K}$ with $\mathrm{GdBaCo}_{2} \mathrm{O}_{5+\delta}$ when $\delta \approx 0.5 .^{1-5}$ However, despite a number of experimental and theoretical studies in layered cobaltites, great controversy has arisen regarding the Co spin state and the microscopic origin of IMT. In the year 2000, Moritomo et al. $^{6}$ suggested that the IMT is induced by a spin-state transition (SST) from an orbital-ordered (OO) IS state to the HS state in both $\mathrm{Co}_{\text {pyr }}$ and $\mathrm{Co}_{\text {oct }}$ sites, basing their consideration on neutron powder diffraction of $\mathrm{TbBaCo}_{2} \mathrm{O}_{5.5}$. Later, a synchrotron radiation x-ray powder diffraction (XRPD) study of $\mathrm{GdBaCo}_{2} \mathrm{O}_{5.5}$ ruled out any $\mathrm{OO}$, suggesting that the IMT should be related to a spin-state switch from LS to HS states at $\mathrm{Co}_{\text {oct }}$. Conversely, the $\mathrm{Co}_{\text {pyr }}$ should remain in IS state at both sides of the IMT. ${ }^{4}$ This is consistent with $a b$ initio calculations that verified the stability of pyramidal IS states in $\operatorname{LnBaCo} \mathrm{O}_{5.5}$ systems. ${ }^{7}$ Maignan et $a l .{ }^{8}$ explained the interplay between IMT and SST using a model based on conversion of HS to LS state in $\mathrm{Co}_{\text {oct }}$ at $T$ $<T_{\mathrm{IM}}$ which would immobilize the electron charge carriers through a "spin blockade" mechanism between $\mathrm{HSCo}^{2+}$ and
$\mathrm{LSCo}^{3+}$. In strong contrast with SST, a muon-spin relaxation study on $\operatorname{Ln} \mathrm{BaCo}_{2} \mathrm{O}_{5+\delta} \delta \approx 0.5$ suggested that the $\mathrm{HS}$ state of $\mathrm{Co}^{3+}$ is retained at $T<300 \mathrm{~K} .{ }^{9}$ Recently thermal expansion measurements on $\mathrm{GdBaCo}_{2} \mathrm{O}_{5.5}$ confirmed the SST as the driving force for IMT excluding the occurrence of stepwise SST at lower temperatures. ${ }^{5}$ Another model based on density functional theory calculations ${ }^{10}$ and supported by photoemission $^{11}$ and isotope-effect neutron diffraction data ${ }^{12}$ suggested that the IMT is due to hole delocalization in the $\mathrm{Co}^{3+}$ HS state rather than to SST. ${ }^{10,12}$

In this paper we present a EPR study on $\mathrm{GdBaCo}_{2} \mathrm{O}_{5+\delta}$ combined with synchrotron XRPD as a function of $\delta$ and of the temperature across the IM transition. EPR spectroscopy allows direct access to the spin-environment interactions through the investigation of the spin relaxation behavior. ${ }^{13}$ The EPR signal markedly changes with increasing temperature for $\delta=0.54(1)$ and $\delta=0.57(1)$ samples, indicating that the spin exchange frequency increases above $T_{\mathrm{IM}}$ in both cases. By comparing EPR and XRPD $T$-dependent experimental results we clearly demonstrate that the interplay between Co spin state and crystal lattice plays a central role in the IM mechanism of this layered cobaltite.

\section{EXPERIMENTAL PROCEDURES AND RESULTS}

$\mathrm{GdBaCo}_{2} \mathrm{O}_{5+\delta}$ was synthesized by a conventional solidstate reaction technique ${ }^{14}$ and the desired oxygen content was adjusted on three aliquots according to annealing conditions and thermal treatments reported by Taskin et al. ${ }^{2}$ The oxygen content was determined by the procedure described in Ref. 15; $\delta=0.54(1), \delta=0.57(1)$, and $\delta=0.63(1)$ were found for the three prepared samples.

XRPD patterns were collected at the ID31 beamline of the European Synchrotron Radiation Facility (ESRF) in Grenoble. For each sample, 40 diffraction patterns were collected in the $0<2 \vartheta<20^{\circ}$ range from 300 to $400 \mathrm{~K}$ selecting a wavelength of $\lambda=0.39620(5) \AA$. Moreover, some high-quality diffraction patterns were collected at $\lambda=0.35422(1) \AA$ for a total counting time of $1 \mathrm{~h}$ at selected $T$ between 300 and 


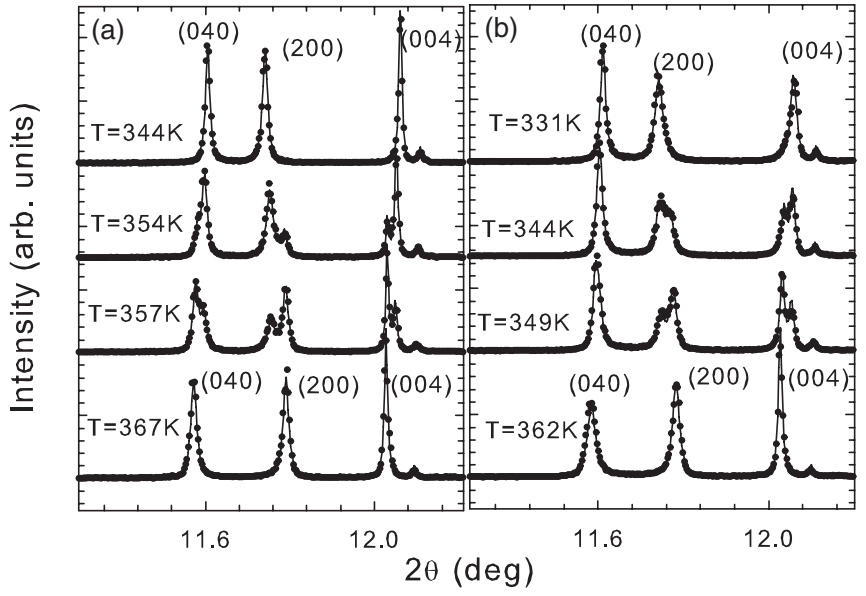

FIG. 1. (a), (b) Selected region of XRPD patterns as collected at different temperatures for the $\delta=0.54(1)$ and $\delta=0.57(1)$ samples, respectively. The black dots are the experimental data while the continuous lines are the results from Rietveld refinements. The Miller indexes of the diffraction peaks are also reported. Typical agreement factor $R\left(F^{2}\right)$ between observed and calculated XRPD patterns range from 0.06 to 0.08 .

$400 \mathrm{~K}$ depending on sample composition. Data were analyzed using the Rietveld method as implemented in GSAS software suite. ${ }^{16}$ All the data sets have been refined against the Pmmm model derived from the cubic perovskite by doubling along the $b$ and $c$ axes $\left(a_{c} \times 2 a_{c} \times 2 a_{c}\right.$ unit cell, where $a_{c}$ stands for the cell parameter of the cubic perovskite lattice). ${ }^{4}$ To account for the actual $\delta$ values in structural models, we located the extra oxygen ions (with respect to $\delta=0.5$ ) at the $1 c(0,0,1 / 2)$ position considering the $1 g(0,1 / 2,1 / 2)$ fully occupied. This is equivalent to considering an ordered alternation of $\mathrm{Co}_{\mathrm{pyr}}$ and $\mathrm{Co}_{\text {oct }}$ along the [010] direction for $\delta=0.5 .^{1,4,6,17}$ The Rietveld refinements in selected portions of the diffraction patterns collected at various temperatures for $\delta=0.54(1)$ and $\delta=0.57(1)$ samples are shown in Fig. 1. Refined lattice parameters are shown for all the samples in Fig. 2.

The presence of splitting in the diffraction profile of (040), (020), and (002) peaks is evident for $\delta=0.54(1)$ and $\delta=$ $0.57(1)$ in a narrow $T$ range $(\sim 7-8 \mathrm{~K})$ below $T_{\mathrm{IM}} \approx 365 \mathrm{~K}^{1-5}$ as shown in Fig. 1. This phase transition in the proximity of IMT is well known to occur in $\operatorname{Ln} \mathrm{BaCo}_{2} \mathrm{O}_{5.5}{ }^{4,6,12,18}$ and the presence of peak splitting in the XRPD patterns is related to coexistence of the low- and high- $T$ structural phases. The latter finding provides evidence for the first order of the transition. ${ }^{18}$ As shown in Fig. 2, with increasing $T$ toward IMT, both the $b$ and $c$ lattice parameters exhibit a steplike increase while $a$ suddenly shrinks for both $\delta=0.54(1)$ and $\delta=0.57(1)$ samples. No phase coexistence was evidenced by the XRPD patterns of the $\delta=0.63(1)$ sample, since $b$ and $c$ linearly increase in $300 \leqslant T \leqslant 400 \mathrm{~K}$ range and $a$ decreases up to $T \sim 325 \mathrm{~K}$. The anisotropic thermal expansion of the unit cell parameters results in a variation of the unit cell volume. By comparing unit cell volume values as a function of $T$ and $\delta$ reported in the insets of Fig. 2, we note that the discontinuity which holds for first-order transition is apparent only for $\delta=0.54(1)$. This provides an indication that the order of the structural phase

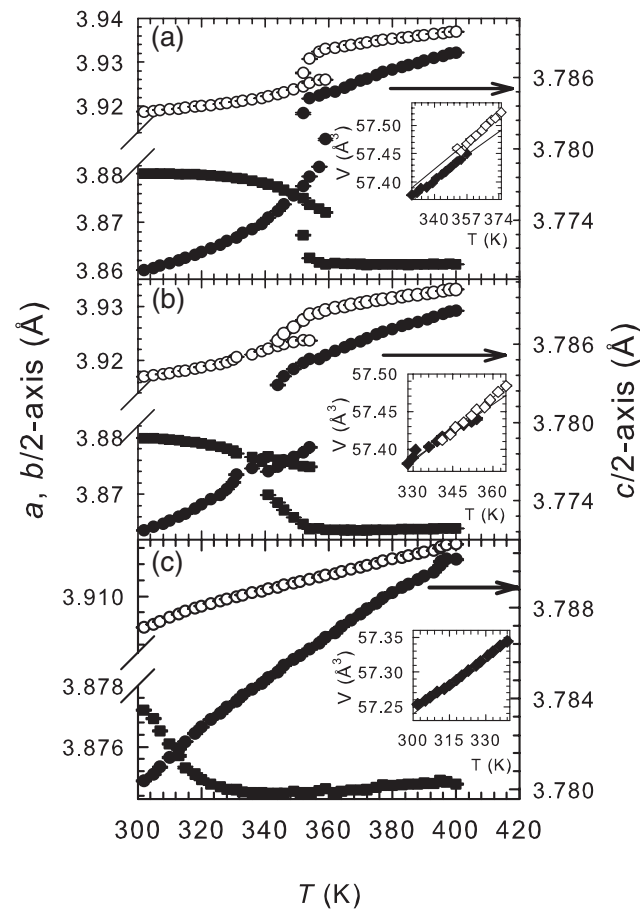

FIG. 2. (a)-(c) Lattice parameters $a$ (filled squares), $b / 2$ (empty circles), and $c / 2$ (filled circles) as a function of temperature are displayed for $\delta=0.54(1), 0.57(1)$, and 0.63(1), respectively. Insets: the temperature dependence of the unit cell volume for selected temperature range.

transition may change from the first to second order upon increasing $\delta$.

EPR measurements were performed on a Bruker ELEXSYS spectrometer equipped with an ER4102ST standard rectangular cavity at $X$ band $(9.4 \mathrm{GHz})$ frequency in the temperature range $305-450 \mathrm{~K}$ every $5 \mathrm{~K}$. Powdered samples were placed into quartz tubes and the derivative $d P / d H$ of power $P$ absorbed was recorded as a function of the static magnetic field $H$.

In Fig. 3 we show EPR spectra as a function of temperature for $\delta=0.54(1)$ sample, as an example. The signal consists of a single broad resonance line. It is well known that the $\mathrm{Co}^{3+}$ EPR signal cannot be observed because of a too-short relaxation time ${ }^{19}$ so that the EPR signals in $\mathrm{GdBaCo}_{2} \mathrm{O}_{5+\delta}$ can be originated from the shift and/or broadening of the $\mathrm{Gd}^{3+}$ resonance caused by exchange interaction $J_{f s} S \cdot s$ between localized $4 f$ electron spin $(S)$ and the spins $(s)$ of the transition metal. ${ }^{13}$

At each $T$, the spectra were well fitted by a single Dysonian lineshape ${ }^{20}$ shown as a solid line in the inset of Fig. 3. However, meaningless negative dispersion-to-absorption $\alpha$ contributions were evaluated in these EPR features. By comparing our spectra with the Dysonian line reported in the literature for other perovskitic systems, ${ }^{21}$ we found that the EPR features measured by us correspond only to a part of the literature-reported line. ${ }^{21}$ In particular, the left lobe of the EPR spectrum shown in the inset of Fig. 3 is too broad to be fully observable. This leads to unreliable numerical values of the $x_{0}$ (peak position), $\alpha$, or $w$ (linewidth) parameters. Another possible reason could be the presence of multiple 


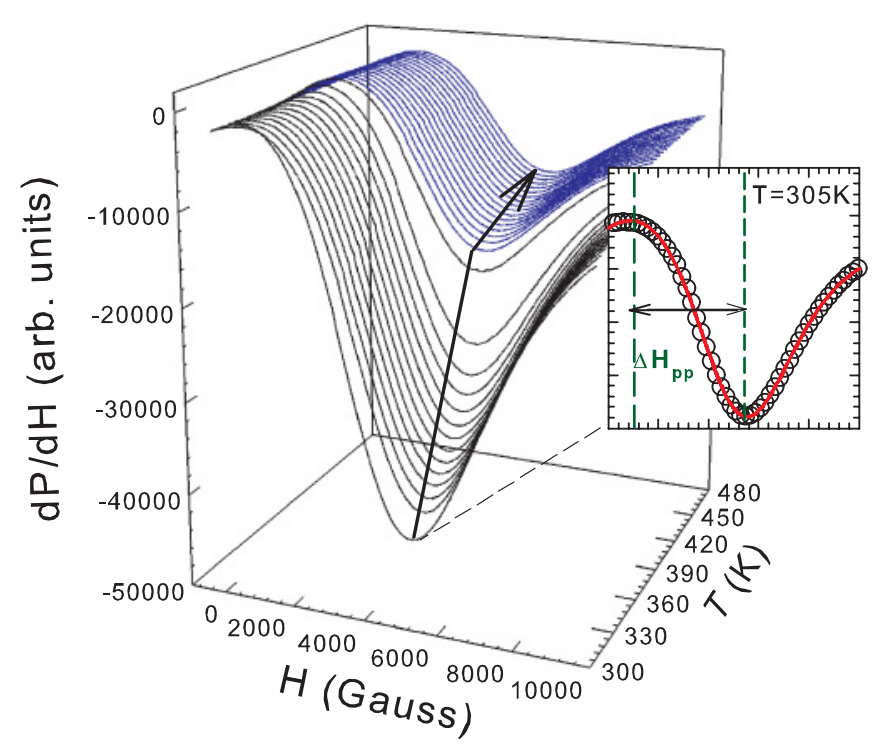

FIG. 3. (Color online) EPR spectra of the $\delta=0.54(1)$ sample as a function of temperature. The arrow outlines their trend with increasing temperature and in particular at the critical temperature $T_{c} \approx 365 \mathrm{~K}$. In the inset an example of fit using a Dysonian line shape is shown (open circles: observed data; solid line: fit).

signals as reported for other Co-based oxides. ${ }^{22}$ However, attempts by linear combinations of Lorentzian and Gaussian functions gave no improvement to the lineshape description. We decided to extract the peak-to-peak linewidth $\left(\Delta H_{\mathrm{pp}}\right)$ by direct observation of the experimental patterns as indicated in the inset of Fig. 3. The trend of $\Delta H_{\mathrm{pp}}$ with temperature is shown in Figs. 4(a)-4(c) for all the samples. It should be noted that in our spectra the base line is not well defined and, hence, we cannot determine directly the peak position.

$\Delta H_{\mathrm{pp}}$ shows three different temperature-dependent regions for $\delta=0.54(1)$ and $\delta=0.57(1)$ : (i) It is roughly constant or decreases smoothly between 300 and $330 \mathrm{~K}$; (ii) it decreases steeply with increasing $T$ toward a critical temperature $T_{c}$ for $\sim 330<T<\sim 360 \mathrm{~K}$; (iii) it exhibits a distinct kink at $T_{c}=$ $360 \pm 5 \mathrm{~K}$, then approaches constant values up to $T=450 \mathrm{~K}$. The behavior of $\Delta H_{\mathrm{pp}}$ is rather different for the $\delta=0.63(1)$ sample, showing a smaller $\Delta H_{\mathrm{pp}}$ value at $T=300 \mathrm{~K}$ with just a weak decrease at $T_{c} \sim 325 \mathrm{~K}$. All the samples display a similar $\Delta H_{\mathrm{pp}}$ value at $T>T_{c}$. In Fig. 4(d) we compare the $T_{c}$ with the $T_{\mathrm{IM}}$ values determined by Taskin et al. for $\delta=$ 0.50 and $\delta=0.65$ from resistivity measurements. ${ }^{2}$ The good agreement between the two critical temperatures as a function of $\delta$ indicates that the EPR linewidth change at $T_{\mathrm{c}}$ is consistent with the IM transition. $T_{c}=T_{\mathrm{IM}}$ will be considered hereinafter.

\section{DISCUSSION AND CONCLUSION}

According to magnetization measurements performed by Respaud et al. ${ }^{17}$ on a $\mathrm{GdBaCo}_{2} \mathrm{O}_{5.54(3)}$ sample, a drastic change in the Weiss-Curie temperature $\Theta_{\mathrm{WC}}$ and effective cobalt magnetic moment was observed above $T_{\mathrm{IM}}$. In order to estimate the strength of the magnetic interactions involved, we calculated the value of the isotropic exchange constants $(J)$
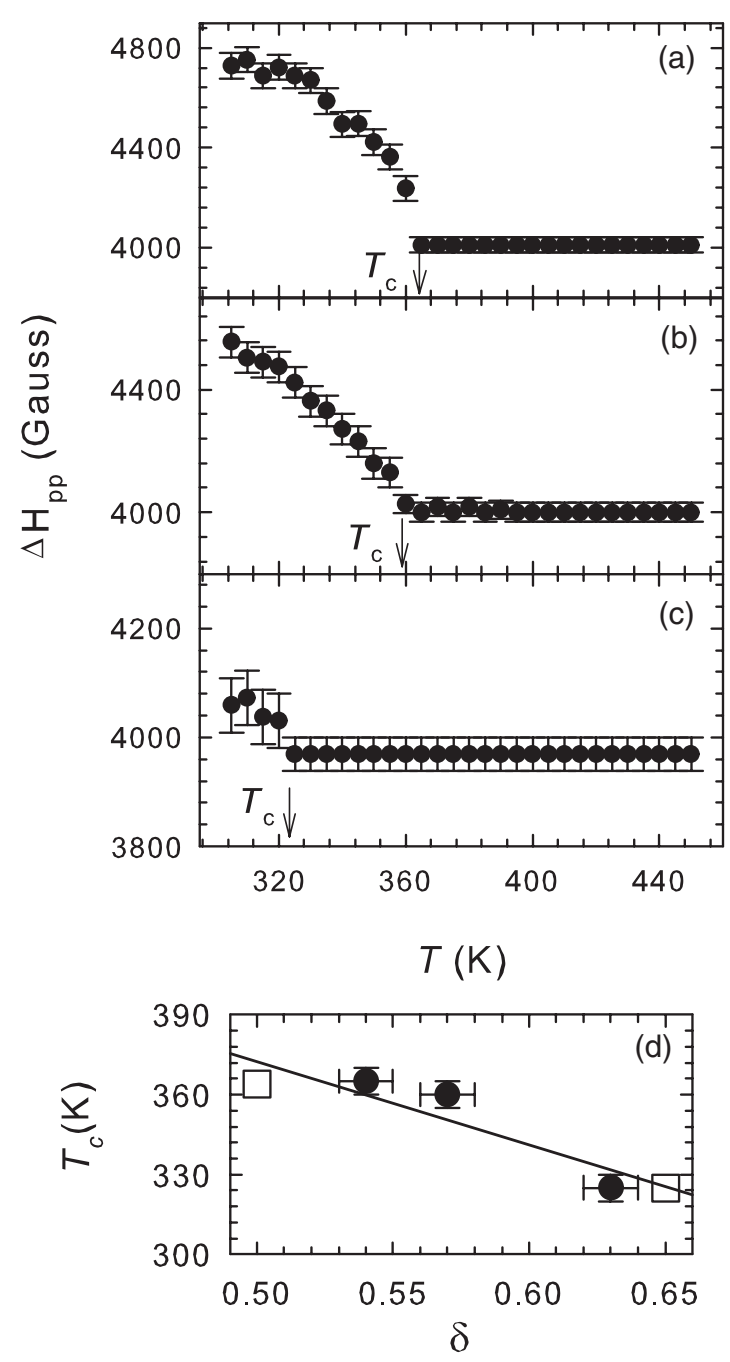

FIG. 4. (a)-(c) $\Delta H_{\mathrm{pp}}$ against temperature for $\delta=0.54(1), \delta=$ $0.57(1)$, and $\delta=0.63(1)$, respectively. (d) The $T_{c}$ (filled circles) highlighted in panels (a)-(c) are compared with $T_{\mathrm{IM}}$ reported by Taskin et al. (Ref. 2) (open squares).

using the molecular field theory for three-dimensional (3D) systems. To compute $J$ from the molecular field equation, ${ }^{23}$ we assumed the model proposed by Taskin et al. ${ }^{1,2}$ in which the magnetic interactions are generated by IS- $\mathrm{Co}^{3+}$ and we used the modulus of the values of $\Theta_{\mathrm{WC}}$ reported below and above the IMT. ${ }^{17}$ By considering $S=1$ for IS- $\mathrm{Co}^{3+}$ and $Z=$ 3 , i.e., by counting only for nearest neighbors, we estimated $\left|J_{\text {ins }}\right| / k_{B} \approx 69 \mathrm{~K}$ and $\left|J_{\text {met }}\right| / k_{B} \approx 150 \mathrm{~K}$ in the insulating and metallic phases, respectively. This corresponds to a ratio of $\left|J_{\text {met }}\right| /\left|J_{\text {ins }}\right| \sim 2$ indicating that the isotropic exchange interactions between cobalt ions increase above $T_{\mathrm{IM}}$.

In the case of strong isotropic exchange interactions the Gaussian EPR linewidth is narrowed into a Lorenztian line with $\Delta H_{\mathrm{pp}}$ given by ${ }^{23-25}$

$$
\Delta H_{\mathrm{pp}}=\frac{\hbar M_{2}}{g \mu_{B} \omega_{\mathrm{ex}}},
$$

where $\omega_{\mathrm{ex}} \approx J / \hbar$ is the frequency of the isotropic exchange interactions caused by the Heisenberg Hamiltonian $H_{\mathrm{ex}}^{i j}$ $\left(=J s_{i} \cdot s_{j}\right)$ between neighboring spins $s_{i}$ and $s_{j}$. It should be 
noted that, in the case of an asymptotic regime as observed at $T \rightarrow 300 \mathrm{~K}$ and at $T \rightarrow T_{\mathrm{IM}}$ for $\delta=0.54(1)$ and $\delta=$ $0.57(1)$ samples, both the second moment $M_{2}$ and the exchange frequency $\omega_{\text {ex }}$ are temperature independent. Basically, Eq. (1) can be employed to explain that the $\Delta H_{\mathrm{pp}}$ asymptotic value at $T>T_{\mathrm{IM}}$ in the metallic phase is smaller than that reached at $T \rightarrow 300 \mathrm{~K}$ in the insulating phase. According to the equation, above $T_{\mathrm{IM}}$ the decreasing of $\Delta H_{\mathrm{pp}}$ can be justified by an increase of the frequency of the isotropic exchange interactions $\omega_{\mathrm{ex}}$ and/or by a decrease in the second moment $M_{2}$. As a matter of fact, $M_{2}$ parameter depends on anisotropic exchange interactions and can be determined by measuring the angular dependence of EPR linewidth or $g$ factor as a function of several orientations of a single crystal sample in the external magnetic field. ${ }^{26}$ As we deal with powdered samples, we cannot resolve the anisotropy of the EPR spectra and, hence, we cannot say anything about the influence of such parameter to explain the strong narrowing. On the other hand, from $\omega_{\mathrm{ex}} \approx J / \hbar$ we can scale the $J$ ratio accordingly and deduce that $\omega_{\mathrm{ex}}^{\mathrm{met}}>\omega_{\mathrm{ex}}^{\mathrm{ins}}$. Thus, the observed temperature decreasing of the $\Delta H_{\mathrm{pp}}$ above $T_{\mathrm{IM}}$ is compatible with an enhancement of the isotropic exchange frequency term in the metallic phase.

According to the model proposed by Maignan et al., ${ }^{8}$ the increase of spin-spin exchange frequency observed at $T>$ $T_{\mathrm{IM}}$ in $\delta=0.54(1)$ and $\delta=0.57(1)$ samples can be easily understood as an increasing of hopping probability of $3 d$ electrons between nearest-neighbor Co ions. The metallic state is interpreted as the motion of an extra electron from an excited $\mathrm{HSCo}^{2+}$ ion to a HS or to an $\mathrm{ISCo}^{3+}$ ion. ${ }^{8}$ However, this model does not explain how $\mathrm{Co}^{2+}$ ions are created and does not consider that a motion of one $\mathrm{e}_{\mathrm{g}}$ electron from $\mathrm{HSCo}^{3+}$ to $\mathrm{ISCo}^{3+}$ would generate an unstable non- $\mathrm{LSCo}^{4+}$. To avoid the production of such cobalt species, we suppose that the $\mathrm{Co}_{\text {oct }}^{3+}$ sites in the metallic phase of an ideal $\delta=0.5$ sample display the IS state together with a $T$-independent $\mathrm{ISCo}_{\mathrm{pyr}}^{3+}{ }^{3+} \mathrm{It}$ should be noted that an $e_{g}$ electron hopping from an $\mathrm{ISCo}_{\mathrm{oct}}^{3+}$ to the next $\mathrm{ISCo}_{\text {oct }}^{3+}$ along the $a$ and $c$ axes and/or to the next $\mathrm{ISCo}_{\mathrm{pyr}}^{3+}$ along the $b$ axis generates, in any case, a couple of $\mathrm{LSCo}^{4+}$ and $\mathrm{HSCo}^{2+}$ stable species. For example, the electron transfer along the $b$ axis from $\mathrm{Co}_{\mathrm{pyr}}$ to $\mathrm{Co}_{\text {oct }}$ and vice versa can be sketched as $t_{2 g}^{5} e_{g}^{1}-\mathrm{ISCo}_{\mathrm{pyr}}^{3+}+t_{2 g}^{5} e_{g}^{1}-\mathrm{ISCo}_{\mathrm{oct}}^{3+} \rightarrow t_{2 g}^{5} e_{g}^{0}-$ $\mathrm{LSCo}_{\mathrm{pyr}}^{4+}+t_{2 g}^{5} e_{g}^{2}-\mathrm{HSCo}_{\mathrm{oct}}^{2+}$ (Fig. 5). This process justifies high $\omega_{\text {ex }}$ above $T_{\mathrm{IM}}$ since the presence of $\mathrm{LSCo}_{\mathrm{pyr}}^{4+}$ and $\mathrm{HSCo}_{\mathrm{oct}}^{2+}$ implies double exchange (DE) interaction between $\mathrm{LSCo}_{\mathrm{pyr}}^{4+}$ and $\mathrm{HSCo}_{\mathrm{oct}}^{2+}$ and $\mathrm{ISCo}_{\mathrm{oct} / \mathrm{pyr}}^{3+}$ ions occurring along the $b$ axis. At the same time, the formation of $e_{g}$ conduction band of IS character gives rise to the observed metallic ground state since DE paths formed by the 3D network of $\mathrm{ISCo}_{\mathrm{oct} / \mathrm{pyr}}^{3+}$ account for electronic delocalization in all directions. Upon cooling below $T_{\text {IM }}$, a SST from IS to LS state can occur solely at $\mathrm{Co}_{\text {oct }}^{3+}$ while the $\mathrm{Co}^{3+}$ in pyramid remains in IS state. ${ }^{4}$ Within this spin configuration, $t_{2 g}$ electron hopping from $\mathrm{LSCo}_{\mathrm{oct}}^{3+}$ to $\mathrm{ISCo}_{\mathrm{pyr}}^{3+}$ produces a couple of $\mathrm{LSCo}_{\mathrm{oct}}^{4+}$ and $\mathrm{HSCo}_{\mathrm{pyr}}^{2+}$. Conversely $e_{g}$ electron hopping from $\mathrm{ISCo}_{\mathrm{pyr}}^{3+}$ to $\mathrm{LSCo}_{\mathrm{oct}}^{3+}$ ions generates stable $\mathrm{LSCo}_{\text {pyr }}^{4+}$ but unstable $\mathrm{ISCO}_{\text {oct }}^{2+}$ (Fig. 5). This latter process accounts for the decreased $\omega_{\text {ex }}$ value since the above sketched model to produce $\mathrm{Co}^{4+}-\mathrm{Co}^{2+}$ pairs would be no longer valid.

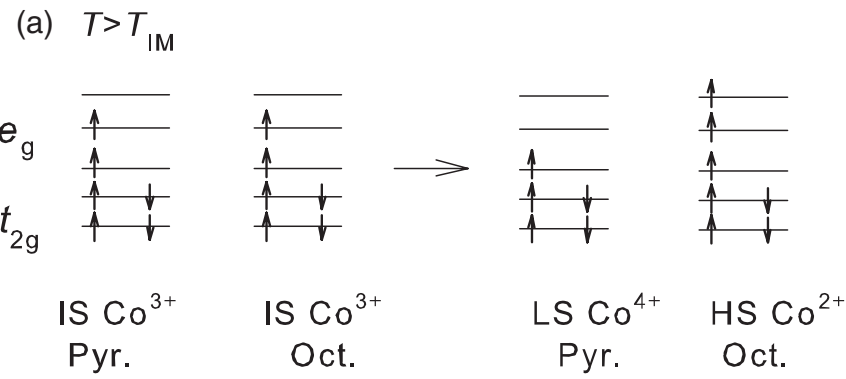

(b) $T<T_{\text {IM }}$
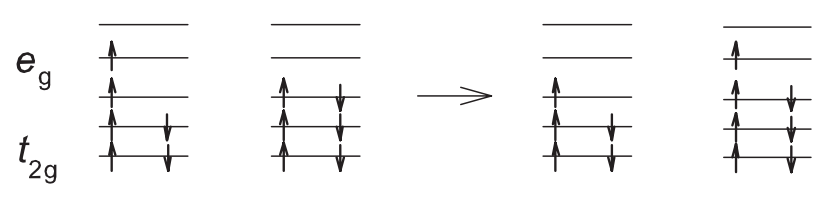

IS $\mathrm{Co}^{3+}$

Pyr.
$\mathrm{LS} \mathrm{Co}^{3+}$
Oct.

IS $\mathrm{Co}^{2+}$ Oct.

FIG. 5. Schematic electronic level diagram of $\mathrm{Co}$ in $\mathrm{GdBaCo}_{2} \mathrm{O}_{5.5}$ showing the processes of $e_{g}$ electron hopping. On the left-hand side of (a), (b) the $e_{g}$ electron transfers from pyramidal to octahedra Co sites are displayed in the metallic and insulating phases, respectively. On the right-hand side of the same panels the diagram related to cobalt species generated after the hopping processes are shown.

Hence, we can say that the proposed mechanism identifies the SST from $\mathrm{LSCo}_{\text {oct }}^{3+}$ to $\mathrm{ISCo}_{\text {oct }}^{3+}$ as a possible origin for the IMT. The temperature-induced SST is corroborated by the sudden unit cell volume expansion observed for $\delta=0.54$ (1) sample. Indeed the transition to a higher spin state in $\mathrm{Co}^{3+}$ implies bigger ionic radius with respect to $\mathrm{LSCo}^{3+} .4,5$

Dealing with $\delta>0.5$ samples, the role of the increased amount of $\mathrm{LSCo}^{4+}$ induced by increasing $\delta$ must be taken into account in the above mechanism. As suggested by Taskin et al. ${ }^{2,3}$ the presence of $\mathrm{LSCo}^{4+}$ can imply double exchange (DE) interaction between $\mathrm{LSCo}^{4+}, \mathrm{ISCo}^{3+}$, and $\mathrm{LSCo}^{3+}$. These exchange channels are active even at room temperature and can be considered to enlighten the gradual improvement in the conductivity that blurs the IMT with increasing $\delta .2,3$ We argue that the $\delta$ dependence of EPR results reported here can be understood considering the interplay between SST and DE. Looking at the $\Delta H_{\mathrm{pp}}$ temperature dependences, distinct transitions were found for $\delta=0.54(1)$ and $\delta=0.57(1)$ samples and we can suggest that the DE contribution is negligible for $\delta \leqslant 0.57$ (i.e., $\mathrm{Co}^{4+} / \mathrm{Co}^{3+} \leqslant 7.5 \%$ ). Conversely, the weak effect observed in the temperature dependence of $\Delta H_{\mathrm{pp}}$ for $\delta=$ $0.63(1)$ (i.e., $\mathrm{Co}^{4+} / \mathrm{Co}^{3+} \approx 15 \%$ ) can be explained considering a strong contribution of DE that accounts for high $\omega_{\mathrm{ex}}$ value even at room temperature. Moreover we observe a gradual decrease of $\Delta H_{\mathrm{pp}}$ value increasing $\delta$ above $0.54(1)$ at $305 \mathrm{~K}$. This is a further confirmation that the narrowing of the EPR line with increasing $\mathrm{Co}^{4+}$ concentration can be fully explained by the $\mathrm{DE}$ channel between $\mathrm{LSCo}^{4+}$ and $\mathrm{LS}^{4} \mathrm{ISCo}^{3+}$ active at $T<T_{\mathrm{IM}}$.

To compare the XRPD and EPR results, in Fig. 6(a) we plot the normalized change of $\Delta H_{\mathrm{pp}}, \delta \Delta H_{\mathrm{pp}} / \Delta H_{\mathrm{pp}}=$ $\left[\Delta H_{\mathrm{pp}}(305)-\Delta H_{\mathrm{pp}}(T)\right] / \Delta H_{\mathrm{pp}}(305)$, together with the normalized changes of the lattice constants for $\delta=0.54(1)$ sample, 


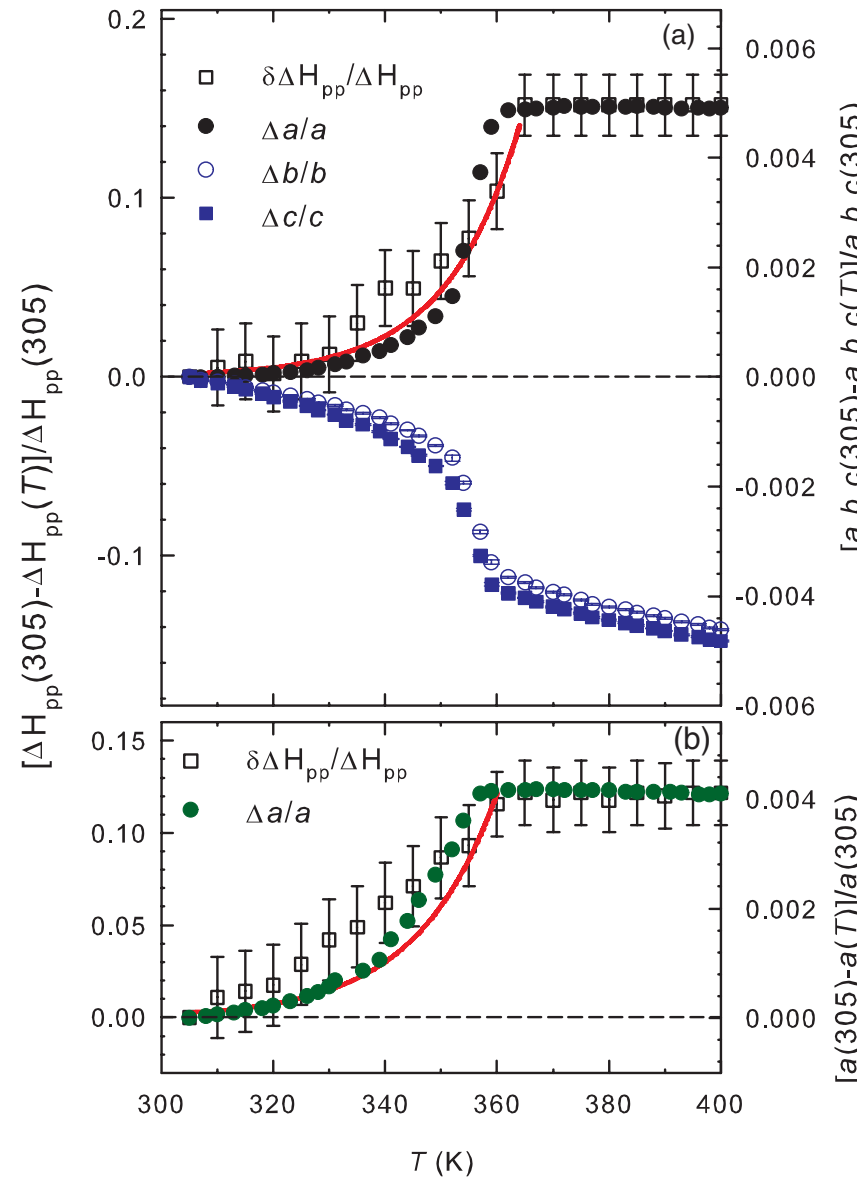

FIG. 6. (Color online) (a) The normalized $\Delta H_{\mathrm{pp}}$ (open squares) values are reported together with the normalized $a$ (filled circles), $b / 2$ (open circles), and $c / 2$ (filled squares) axes for $\delta=0.54(1)$ sample. (b) The normalized data ( $a$ axis and $\Delta H_{\mathrm{pp}}$ ) are reported for $\delta=$ $0.57(1)$. The activation model proposed (solid line) is superimposed in panels (a), (b) to experimental data.

$\Delta L / L=[L(305)-L(T)] / L(305)$ where $L=a, b, c$. In the biphasic region we considered an average lattice parameter value calculated as $F_{1} L_{1}+F_{2} L_{2}$, where $L$ is the lattice parameter and $F$ is the relative phase fraction. Indices 1 and 2 stand for low- $T$ and high- $T$ phases, respectively.

As shown in Fig. 6(a), it is clear that the temperature dependences of $\delta \Delta H_{\mathrm{pp}} / \Delta H_{\mathrm{pp}}$ and $\Delta a / a$ fairly overlap each other and the change of $\Delta H_{\mathrm{pp}}$ is much larger than that of the latter parameter; e.g., at $T=400 \mathrm{~K}$, $\delta \Delta H_{\mathrm{pp}} / \Delta H_{\mathrm{pp}} \approx 15 \%$ and $\Delta a / a \approx 0.5 \%$. In particular, $\Delta a / a$ is the only normalized lattice parameter that increases as a function of $T$ and saturates above $T_{\mathrm{IM}}$ in a manner remarkably similar to $\delta \Delta H_{\mathrm{pp}} / \Delta H_{\mathrm{pp}}$. This could give evidence that the Co-O-Co interatomic distances along the [100] direction contract with increasing $T$ giving rise to the enhancement of the spin-spin exchange frequency along this direction and the decreasing of $\Delta H_{\mathrm{pp}}$. These results suggest spin-lattice interaction along the $a$ axis.

Anisotropic interactions involving $\mathrm{Co}^{3+}$ spin and crystal structure are not new in the $\mathrm{GdBaCo}_{2} \mathrm{O}_{5+\delta}$ system. In particular, Taskin et al. ${ }^{1}$ found Ising-like behavior of spins along the $a$ axis in the ferromagnetic (FM) phase of
$\mathrm{GdBaCo}_{2} \mathrm{O}_{5.5}$ single crystal below the Curie temperature $T_{\text {Curie }}$ $\sim 275 \mathrm{~K} .{ }^{1,2}$ According to the proposed model, ${ }^{1}$ the insulating FM ground state is generated from the interaction of $\mathrm{ISCo}^{3+}$ ions forming two-leg ladders extended along the $a$ axis. ${ }^{1,2}$ These ladders are separated from each other, along the $b$ axis, by ac $\mathrm{CoO}_{2}$ layers composed by nonmagnetic $\mathrm{LSCo}^{3+} .1,2$ Above $T_{\text {Curie }}$ a clear FM-to-paramagnetic (PM) transition is observed in the magnetization curve of $\delta \approx 0.5$ sample composition. ${ }^{1}$ Basically, two contrasting explanations can be given for such transition: (1) Cobalt spins keep their spin easy axis in the direction of the $a$ axis but the interaction between FM ladders becomes weaker than the PM contribution of $\mathrm{Gd}^{3+} ;(2)$ the orientation FM order of $\mathrm{Co}^{3+}$ Ising-like spins is completely destroyed above $T_{\text {Curie }}$.

Ising-like behavior of spins and the observed $\delta \Delta H_{\mathrm{pp}} / \Delta H_{\mathrm{pp}}$ and $\Delta a / a$ scaling behavior shows that the $a$ axis is the preferred crystallographic direction for distinct phenomena related to the FM and the PM phases. According to Taskin et $a{ }^{1,2}$ in the FM phase the $a$ axis is the preferred direction for magnetization which precludes the formation of magnetic moments along the $b$ and $c$ axes. Above $T_{\text {Curie }}$, in the PM phase, the $a$ axis is the preferred direction for the spinlattice interaction which is connected mainly to spin-spin exchange channels. In order to make a link between the two distinct phenomena occurring along the same crystallographic direction, we might suggest that something related to the nature of spins of the FM phase is retained along the $a$ axis even when the phase is PM. This could support explanation (1) given above and, more importantly, the bridge between FM and PM could be useful to figure out the interplay between the spin-lattice interaction and SST. Indeed, with increasing $T$ and by approaching the IMT from the FM phase, the SST from LS to $\mathrm{ISCo}^{3+}$ occurs in the octahedral $a c$ layers stacked along the $b$ axis. On the other side, we suggest that the generation of the $\mathrm{ISCo}^{3+}$ state, holding to lattice deformation, can be significantly influenced by the nature of spins of the FM phase along the $a$ axis. Hence, strong coupling between spin-spin exchange interactions and the $a$ lattice parameter can be made explaining the observed $\Delta a / a$ and $\delta \Delta H_{\mathrm{pp}} / \Delta H_{\mathrm{pp}}$ scaling.

In our model the SST is linked with an increasing of spin-spin exchange frequency which is the driving force of IMT. The formation of the metallic phase could be then explained by thermally activated hopping of spins between neighboring Co sites where the SST from $t_{2 g}^{6} e_{g}^{0}$-LS state to a cobalt high-spin state enhances the number of electrons in the $e_{g}$ conduction band. ${ }^{4}$ To account for such behavior we consider that the change of $\Delta H_{\mathrm{pp}}$ can be described by $\delta \Delta H_{\mathrm{pp}} / \Delta H_{\mathrm{pp}} \approx K \exp \left[-E(T) / k_{\mathrm{B}} T\right]$ where $K$ is a constant and $E(T)$ is a temperature-dependent energy gap. The energy $E(T)$ is given by the parametrized power function $E(T)=$ $E_{0}\left[1-\left(T / T_{\mathrm{IM}}\right)^{3.39}\right]$ used to model the temperature dependence of energy splitting between ground and excited spin states of $\mathrm{Co}$ in $\mathrm{GdCoO}_{3}$ system. ${ }^{27}$ Using the optical band-gap width $E_{\mathrm{g}}=0.26 \mathrm{eV}$ given in Ref. 28, we calculated the solid lines shown in Figs. 6(a) and 6(b). The good agreement between model and observed data proves the role of excited Co spin states and, thus, SST in the IMT dynamics of the $\delta=$ 0.54 (1) sample. These considerations are still valid for the $\delta=$ 0.57(1) sample, as shown in Fig. 6(b). Conversely, just a weak 

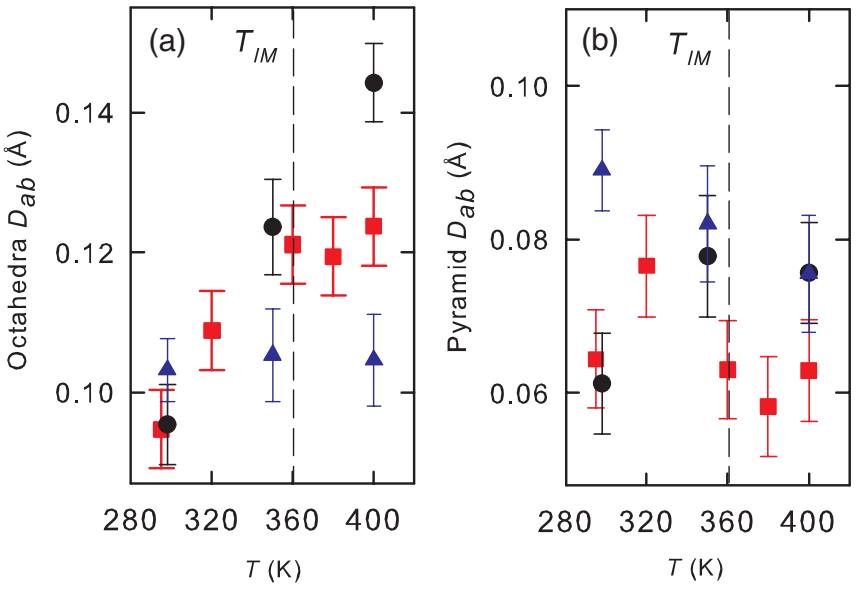

FIG. 7. (Color online) (a), (b) The $a b$ planar bond length difference $\left(D_{a b}\right)$ values as a function of $T$ for $\delta=0.54$ (1) (circles), to $\delta=0.57(1)$ (squares) and $\delta=0.63(1)$ (triangles) are reported in the octahedral and pyramidal environment, respectively.

correlation between the $\Delta a / a$ and $\delta \Delta H_{\mathrm{pp}} / \Delta H_{\mathrm{pp}}$ trends was found for $\delta=0.63(1)$ up to $\sim 325 \mathrm{~K}$.

$\mathrm{Co}^{3+}$ in IS state is a Jahn-Teller (JT) active ion, so that we expect an increased JT distortion across $T_{\mathrm{IM}}$. Aiming to account for $Q_{2}$-type JT distortion, we calculated the differences between $a b$-planar long and short Co-O bond lengths ${ }^{10}\left(D_{a b}\right)$ obtained by Rietveld refinements against the high-quality XRPD data. ${ }^{29}$ In Fig. 7 the $D_{a b}$ values related to octahedra and pyramids are plotted as a function of $T$ for all the samples.

In the former case, $D_{a b}$ increases linearly with increasing temperature for $\delta=0.54(1)$, indicating that the $Q_{2}$-type distortion of the basal plane increases when heating above $T_{\mathrm{IM}}$. Interesting features come out with increasing $\delta$. At $T \approx$ $300 \mathrm{~K}$, the $D_{a b}$ values are very similar for $\delta=0.54(1), \delta=$ $0.57(1)$, and $\delta=0.63(1)$. This indicates that increasing $\delta$ well above 0.5 does not significantly affect the Co-O distances in the octahedra. $D_{a b}$ seems to increase linearly with increasing $T$ for $\delta=0.57(1)$, approaching a constant value above $T_{\mathrm{IM}}$. Conversely, $D_{a b}$ remains practically unchanged for $\delta=$ $0.63(1)$. In the pyramid, the $D_{a b}$ parameters were weakly $\delta$ dependent and they did not change significantly with $T$. This is in agreement with the assumption of $T$-independent $\mathrm{ISCo}_{\mathrm{pyr}}^{3+}$ states

The increasing of $D_{a b}$ distortion observed by the XRPD analysis of the $\delta=0.54$ (1) sample corroborates the occurrence of the proposed SST through the gradual population of the JT-active IS state. On the other hand we have suggested that the DE interactions between $\mathrm{LSCo}^{4+}$ and $\mathrm{LSCo}_{\mathrm{oct}}^{3+}$ and $\mathrm{ISCo}_{\mathrm{pyr}}^{3+}$ (Ref. 1) strengthens with $\delta$ increasing above $\delta \geqslant 0.54(1)$ giving rise to a blurred IMT. ${ }^{2}$ We then expect that the strong DE contribution can also restrain the temperature-induced SST by making the $\mathrm{LSCo}_{\text {oct }}^{3+}$ species less available for the transition. This leaves the high-temperature $\mathrm{ISCo}^{3+}$ states less populated and destroys the JT effect at $\mathrm{Co}_{\text {oct }}^{3+}$ sites. Such effects are supported by the absence of a sudden unit cell volume expansion across IMT and the saturation of $D_{a b}$ above $T_{\mathrm{IM}}$ for $\delta=0.57(1)$ sample as shown in Fig. 7. In addition, the lack of phase coexistence and the temperature-independent behavior of the $D_{a b}$ parameter for $\delta=0.63(1)$ further confirms the removal of the JT with increasing $\delta$. These structural effects give evidence to the occurrence of a transition from low-spin to JT-active intermediate spin state and should support the mechanism proposed to explain IMT.

In conclusion, the increasing of electron hopping probability between $\mathrm{Co}$ in different spin states can be indentified as the main driving force for IMT in $\mathrm{GdBaCo}_{2} \mathrm{O}_{5+\delta}$ system. EPR data are consistent with this model and the increasing of $\omega_{\mathrm{ex}}$ with increasing $T$ can be explained by taking into account the SST from $\mathrm{LSCo}_{\text {oct }}^{3+}$ to $\mathrm{ISCo}_{\mathrm{oct}}^{3+}$. XRPD and EPR results give evidence about a strong coupling between the SST and the crystal structure. To figure out the nature of this coupling and its connection with IMT two ingredients are needed: (i) spin-lattice interaction along the $a$ axis; (ii) JT distortion induced by $\mathrm{ISCo}_{\text {oct }}^{3+}$ which is removed with increasing $\delta$ giving rise to a blurred IMT.

\section{ACKNOWLEDGMENTS}

The authors gratefully acknowledge the European Synchrotron Radiation Facility for provision of beam time and Dr. Adrian Hill for assistance in using the ID31 beamline and an anonymous reviewer for fruitful comments. *mattia.allieta@unimi.it

${ }^{1}$ A. A. Taskin, A. N. Lavrov, and Y. Ando, Phys. Rev. Lett. 90, 227201 (2003).

${ }^{2}$ A. A. Taskin, A. N. Lavrov, and Y. Ando, Phys. Rev. B 71, 134414 (2005).

${ }^{3}$ A. A. Taskin and Y. Ando, Phys. Rev. Lett. 95, 176603 (2005).

${ }^{4}$ C. Frontera, J. L. García-Muñoz, A. Llobet, and M. A. G. Aranda, Phys. Rev. B 65, 180405(R) (2002).

${ }^{5}$ K. R. Zhdanov, M. Yu. Kameneva, L. P. Kozeeva, and A. N. Lavrov, Phys. Solid State 52, 1688 (2010).

${ }^{6}$ Y. Moritomo, T. Akimoto, M. Takeo, A. Machida, E. Nishibori, M. Takata, M. Sakata, K. Ohoyama, and A. Nakamura, Phys. Rev. B 61, 13325(R) (2000).
${ }^{7}$ V. Pardo and D. Baldomir, Phys. Rev. B 73, 165117 (2006).

${ }^{8}$ A. Maignan, V. Caignaert, B. Raveau, D. Khomskii, and G. Sawatzky, Phys. Rev. Lett. 93, 026401 (2004).

${ }^{9}$ H. Luetkens, M. Stingaciu, Yu. G. Pashkevich, K. Conder, E. Pomjakushina, A. A. Gusev, K. V. Lamonova, P. Lemmens, and H.-H. Klauss, Phys. Rev. Lett. 101, 017601 (2008).

${ }^{10} \mathrm{H}$. Wu, J. Phys.: Condens. Matter 15, 503 (2003).

${ }^{11}$ W. R. Flavell, A. G. Thomas, D. Tsoutsou, A. K. Mallick, M. North, E. A. Seddon, C. Cacho, A. E. R. Malins, S. Patel, R. L. Stockbauer, R. L. Kurtz, P. T. Sprunger, S. N. Barilo, S. V. Shiryaev, and G. L. Bychkov, Phys. Rev. B 70, 224427 (2004).

${ }^{12}$ K. Conder, E. Pomjakushina, V. Pomjakushin, M. Stingaciu, S. Struele, and A. Podlesnyak, J. Phys.: Condens. Matter 17, 5813 (2005). 
${ }^{13}$ C. Rettori, S. B. Oseroff, D. Rao, P. G. Pagliuso, G. E. Barberis, J. Sarrao, Z. Fisk, and M. Hundley, Phys. Rev. B 55, 1016 (1997).

${ }^{14}$ L. Lo Presti, M. Allieta, M. Scavini, P. Ghigna, L. Loconte, V. Scagnoli, and M. Brunelli, Phys. Rev. B 84, 104107 (2011).

${ }^{15}$ K. Conder, E. Pomjakushina, A. Soldatov, and E. Mitberg, Mater. Res. Bull. 40, 257 (2005).

${ }^{16}$ A. C. Larson and R. B. Von Dreele, General Structure Analysis System (GSAS), Los Alamos National Laboratory Report No. LAUR 86-748, 2000.

${ }^{17}$ M. Respaud, C. Frontera, J. L. García-Muñoz, Miguel Ángel G. Aranda, B. Raquet, J. M. Broto, H. Rakoto, M. Goiran, A. Llobet, and J. Rodríguez-Carvajal, Phys. Rev. B 64, 214401 (2001).

${ }^{18}$ E. Pomjakushina, K. Conder, and V. Pomjakushin, Phys. Rev. B 73, 113105 (2006).

${ }^{19}$ J. Arai, K. Ozawa, and T. Ishiguro, J. Magn. Magn. Mater. 226-230, 871 (2001).

${ }^{20}$ F. J. Dyson, Phys. Rev. 98, 349 (1955).

${ }^{21}$ J. P. Joshi and S. V. Bhat, J. Magn. Reson. 168, 284 (2004).
${ }^{22}$ N. Tristan, V. Zestrea, G. Behr, R. Klingeler, B. Büchner, H. A. Krug von Nidda, A. Loidl, and V. Tsurkan, Phys. Rev. B 77, 094412 (2008).

${ }^{23}$ P. W. Anderson and P. R. Weiss, Rev. Mod. Phys. 25269 (1953).

${ }^{24}$ R. Kubo and K. Tomita, J. Phys. Soc. Jpn. 9, 888 (1954).

${ }^{25}$ D. Zakharov et al., in Quantum Magnetism, edited by B. Barbara, Y. Imry, G. Sawatzky, and P. C. E. Stamp (Springer, Berlin, 2008), p. 212.

${ }^{26}$ M. Heinrich, H.-A. Krug von Nidda, R. M. Eremina, A. Loidl, Ch. Helbig, G. Obermeier, and S. Horn, Phys. Rev. Lett. 93, 116402 (2004).

${ }^{27}$ K. Knízek, Z. Jirák, J. Hejtmánek, M. Veverka, M. Marysko, G. Maris, and T. T. M. Palstra, Eur. Phys. J. B. 47, 213 (2005).

${ }^{28}$ A. A. Makhnev, L. V. Nomerovannaya, S. V. Strel'tsov, V. I. Anisimov, S. N. Barilo, and S. V. Shiryaev, Phys. Solid State 51, 525 (2009).

${ }^{29}$ See Supplemental Material at http://link.aps.org/supplemental/ 10.1103/PhysRevB.84.235144 for diffraction patterns and tables containing refined structural parameters obtained from the highquality XRPD collected at $\lambda=0.35422(1) \AA$ at selected temperatures between 300 and $400 \mathrm{~K}$. 